As a first step in defining the molecular cues that may be important for reinnervation of long-term denervated muscle, single adult rat muscle fibers that had been denervated from 2 to 24 months in vivo were maintained in culture for 5 days. Embryonic ventral spinal cord explants were added to some of these cultures. Interactions of neurites with individual short-term (up to 5 months) and long-term (17-24 months) denervated muscle fibers were compared with neurite interactions in cultures of young adult muscle fibers (from 3 to 5-month-old rats) or aged muscle fibers (from 17 to 26month-old rats). We found the following. (1) Three molecules that are found at the neuromuscular junction (NMJ) - acetylcholinesterase (AChE), acetylcholine receptors (AChRs), and gelasmin (an acetylcholine receptor clustering factor that is found enriched at NMJ of adult muscle)-were reduced with increasing periods of denervation but not with aging. (2) The number of neurite contacts at junctional regions of muscle fibers that were formed and maintained on cultured muscle fibers depended on denervation time of the muscle in vivo; very few contacts were made or maintained on long-term denervated fibers. (3) Gelasmin, but not AChE or AChRs, was found at points of neurite contact on all muscle fibers examined, raising the possibility that it may serve as a cue for reinnervation and that its loss from longterm denervated muscle may be, at least in part, involved in the failure of neurite contacts to be made or maintained in culture and possibly in vivo.

Key words: muscle fiber $\bullet$ culture $\bullet$ denervation $\bullet$ gelasmin $\bullet$ acetylcholinesterase $\bullet$ acetylcholine receptors

MUSCLE \& NERVE 12:981-992

1989

\title{
DENERVATED SINGLE MYOFIBERS: NEURITE INTERACTIONS AND SYNAPTIC MOLECULES
}

\author{
JEANNE C. JAY, PhD, and KATE F. BARALD, PhD
}

After nerves innervating skeletal muscle have been severed in vivo, muscle fibers can be reinnervated. ${ }^{5,10,14}$ Considerable interest has focused on the way in which nerves reinnervate

From the Department of Anatomy and Cell Biology, Program in Neuroscience, University of Michigan Medical School. Ann Arbor, MI.

Dr. Jay's present address is the Department of Cell Biology and Anatomy, University of Alabama School of Medicine, Birmingham, AL.

Acknowledgments: This work was supported by grants from the $\mathrm{NIH}$ (NS17017) (subproject), the NSF (PCM-8317271), MDA, Phoenix Foundation, and the Rackham Foundation to Dr. Barald. Dr. Jay's work was supported by a Rackham predoctoral fellowship and by training grant HD07274 from NICHHD. We thank Drs. B.M. Carlson and F. HansenSmith for giving us the feet of animals whose limb muscles had been denervated for other experiments and verifying that muscles were denervated priar to cuiture. Drs. C. Carter-Su, B.M. Carlson, J. Schwartz, D. Thomas, and $\mathrm{G}$. Brewer provided feet from young and aged control animals. We thank Drs. B.M. Carlson, C. Carter-Su, A.L. Dunathan, T.J. Hinterberger, and K.W. Tosney for comments on the manuscript. L.A. Polacek provided expert technical assistance. S.L. Moskwiak, N. Smith, F. Gruda, and P.B. Jewelt expertly prepared the typescript.

Address reprint requests to Dr. Barald at the Department of Anatomy and Cell Biology, University of Michigan Medical School, Box 0616. Ann Arbor. Ml 48109 .

Accepted for publication December 7,1988

$0148 \cdot 639 \times / 1212 / 0981 \$ 04.0011 ?$

(6) 1989 John Wiley \& Sons Inc. muscle after short times (days or weeks) without neural input (for a review, see Sanes and Covault $^{21}$ ). However, after injury or in disease states, muscle may be denervated for many months or perhaps years. In such cases, factors unrelated to the properties of the muscle itself, including the degree of axon regeneration and the amount of connective tissue that is present, play a role in determining whether muscle and nerve will reestablish connections. ${ }^{25}$ The condition of the muscle fiber itself, including atrophic changes and the deleterious effects of inactivity, are, of course, also critical in determining whether and where nerves will form new synapses.

Early studics of rabbit peroneal muscles showed that progressively longer periods of denervation were related to altered patterns of reinnervation and to reduction in functional recovery. ${ }^{10}$ When rabbit peroneal muscles were denervated for 1 month or less, $80 \%$ of the original endplates were reinnervated. However, in these studies, after progressively longer periods of denervation ( 5 months), only $25 \%$ of the original 
endplates were contacted, and ectopic synapses were also found. The ratio of reinnervated original endplates to ectopic synapses was not given in this account. The relative importance of several factors that might have contributed to the success or failure of reinnervation was not assessed. Atrophic or inactivity-induced changes in muscle fibers were not considered separately from variables such as increased amounts of connective and adipose tissue that might physically block nerve access. Furthermore, no attempt was made to identify age-related alterations that might change the susceptibility of muscle to reinnervation. The molecular cues that may be important for reinnervation of denervated muscle are thus, as yet, unidentified.

Studies of denervation and reinnervation at the molecular and cellular levels in vivo are hampered because the processes cannot be monitored closely and continuously over time and because the system is difficult to manipulate experimentally. In the study described in this report, we demonstrate that a tissue culture system provides a useful experimental model for the study of molecules that may be important for reinnervation of young adult, aged, and both short- and long-term denervated individual muscle fibers. Such a system provides an initial means of assessment of molecules that might be involved in reinnervation, and a process for selection of those whose functional role in reinnervation can then be tested in vivo.

\section{MATERIALS AND METHODS}

Short- and Long-Term Denervated Fibers. Protocols for dissociation and preparation of cultures of aged and long-term denervated flexor digitorum brevis (FDB) muscles of adult rats with or without embryonic spinal cord explants were the same as those used for young adult muscle fibers. ${ }^{11}$ Briefly, single muscle fibers were obtained by dissociating muscle in a collagenase solution $(600 \mathrm{U} /$ $\mathrm{ml}$ ) in Dulbecco's phosphate-buffered saline (PBS). PBS contained $2.7 \mathrm{~m} M \mathrm{KCl}, 1.2 \mathrm{~m} M$ $\mathrm{KH}_{2} \mathrm{PO}_{4}, 137 \mathrm{~m} M \mathrm{NaCl}, 8.1 \mathrm{~m} M \quad \mathrm{Na}_{2} \mathrm{HPO}_{4} \cdot 7$ $\mathrm{H}_{2} \mathrm{O}, 0.72 \mathrm{~m} M \mathrm{MgSO}_{4}, 0.16 \mathrm{~m} M \mathrm{CaCl}_{2}$, and 49.4 $\mathrm{m} M$ glucose. Muscle fibers were then plated in a Vitrogen gel (Flow Labs, McLean, VA). ${ }^{11}$ Spinal cord explants were obtained from the ventral halves of spinal cords of 13-14-day-old embryonic rats and were cultured for $2-5$ days. ${ }^{11}$ The initial neurite outgrowth was excised, and explant cores were transferred to 1-day-old muscle fiber cultures. ${ }^{11}$
Denervation In Vivo. Flexor digitorum brevis (FDR) muscles denervated in vivo were obtained from Sprague Dawley rats (Charles River, Indianapolis, IN) in which the sciatic nerve had been severed for as short a time as 2 months or as long as 24 months. FDBs were denoted "short- or longterm denervated" to differentiate them from FDBs removed (and therefore denervated) immediately prior to culture from either young adult or aged rats. For leg muscles denervated from 2 to 7 months (short-term denervated), denervation involved severing the sciatic nerve at the sciatic notch in male Sprague-Dawley rats $(175-250 \mathrm{~g})$. Approximately $1 \mathrm{~cm}$ of the nerve was removed from the distal stump. The proximal stump was reflected and sutured into the skin. Denervation was either bilateral or unilateral. In cases where one leg was denervated, FDB fibers from the contralateral innervated leg were placed in culture as age-matched controls. Since regenerating nerves might have entered the distal nerve sheath, limb muscles were tested for possible reinnervation immediately before removal of muscles. The remainder of the sciatic nerve distal to the level of denervation was stimulated, and lower limb muscles were examined for any signs of contraction. Muscles that showed contractile activity were discarded. After behavioral observations were completed, one or more muscles were removed from the anterior and posterior compartments.

Frozen unfixed sections were stained for the presence of nerves; absence of nerves was noted in all muscles taken from rats used in these studies. For leg muscles denervated for 24 months, the sciatic nerve in male Wistar-Fisher 455 rats (from the rat colony, Department of Biology, University of Michigan) was cut in two places: at the level of the sciatic notch and more distally; $2.5 \mathrm{~cm}$ of nerve was removed from between the cuts. The proximal stump was sutured and implanted into a hip muscle; the distal stump was also sutured to prevent possible reentry of nerves. Nerve stimulation of the leg which contained denervated muscle was done 2 months prior to sacrifice of the animal. At the time no indication of leg movement was noted. All of the experiments described in this report were carried out according to the National Research Council Guide for the care and use of laboratory animals and under the veterinary care and supervision of the University of Michigan Unit for Laboratory Animal Medicine in the laboratory of Dr. B. M. Carlson.

Data from short- and long-term denervated 
muscle fibers were divided into 3 groups: (1) fibers assayed from muscle denervated for $2-3$ months and obtained from 5 animals was combined with data from muscle denervated for $4-5$ months from 8 animals, because the data were similar for all muscles denervated from 2 to 5 months; (2) 6-7-month denervated muscle was obtained from 11 animals; (3) 17-24-month denervated muscle was taken from 5 animals.

Fiber Viability. Two methods were used for assessing fiber viability. All cultures were initially stained with $0.1 \%$ trypan blue which is excluded by viable cells, ${ }^{8,16}$ Cultures were examined with a Leitz Diavert microscope equipped with phase contrast optics. Phase contrast criteria included the presence of clear striations and peripherally located nuclei. However, not all muscle fibers that had been denervated for longer than 5 months and that excluded trypan blue met all phase contrast criteria for viability; therefore, phase contrast criteria were used in assessing viability of these fibers.

Localization of Acetylcholine Receptors, Acetylcho. linesterase, and Gelasmin. Cultures were fixed for 30 minutes in Liebowitz (L-15) (GIBCO, Grand Island, NY) medium-based fixative containing $0.15 \%$ glutaraldehyde and $2 \%$ formaldehyde. ${ }^{17}$ After fixation, cultures werc rinsed with PBS (see above) and then with PBS containing $1 \%$ bovine serum albumin (BSA).

Acetylcholine Receptors. To visualize AChRs, cultures were incubated for 1 hour at room temperature with $1 \times 10^{-8} M$ rhodamine-conjugated alpha-bungarotoxin (R-BTX). Cultures were then rinsed with PBS-1\% BSA followed by PBS. Fibers were examincd for R-B TX binding sites, with the use of an oil immersion (63x) objective, on a Leitz Diavert microscope equipped for incident illumination with narrow-band filter combinations selective for tetramethylrhodamine or fluorescein isothiocyanate (TRITC or FITC; Vector Labs, Burlingame, CA, or Sigma, St. Iouis, MO). Photographs were taken using Kodak Ektachrome 400 ASA film; the ASA was set at 200 with a Leitz Vario-Orthomat. Rhodamine alpha-bungarotoxin was prepared according to the method of Ravdin and Axelrod. ${ }^{18}$ Alphabungarotoxin was obtained from Biotoxins, Inc. (St. Cloud, FI.). By noting the location of any given fiber with the stage micrometers, the same fiber could be relocated after application of any other stain or immunoglobulin and then rephotographed.

Acetylcholinesterase. Cultures were treated with a modified Karnovsky stain $^{17}$ to reveal AChE. The staining solution was removed after 5 hours and replaced with PBS. Specific inhibitors of AChE and nonspecific esterase have been used to ensure that only true AChE, was being examined. Fibers were then located by means of a grid, examined for distribution of $\mathrm{AChE}$, and photographed. Viable fibers were viewed with a $50 \times$ oil-immersion objective in an inverted phase contrast/ fluorescence microscope.

Confirmation of the presensce of junctional $\mathrm{AChE}$ and AChRs was made on undissociated aged and short- and long-term denervated muscle. Preparations of fresh muscle were placed on $45 \times 60 \mathrm{~mm}$ cover slips. After the medium was removed, fibers were stained either with R-BTX to reveal AChRs or with a modified Karnovsky stain for AChE activity. ${ }^{17}$

Gelasmin. A monoclonal antibody to gelasmin, SBL-1, ${ }^{1,2}$ was used at dilutions of $1 / 500$ to label cryostat sections of FDB muscle as well as cultured FDB fibers. Rhodamine-bungarotoxin $\left(10^{-8} M\right)$ was used simultaneously to stain AChR at neuromuscular junctions (NMJs) some of which had been previously stained for AChE activity. After extensive washing in PBS containing $1 \%$ BSA, a second step rabbit antimouse IgG conjugated with fluorescein at 1/100 (Cappel Labs, Malvern, PA) was used. Controls, including a monoclonal antibody to non-endplate basal lamina called EJBL-1 $1^{1,2}$ as well as omission of the primary and secondary antibodies, were also performed. Both freshly dissected FDB muscles and cultured individual muscle fibers were stained.

Determination of Frequency of Neurite Association With Junctional and Extrajunctional Regions of Muscle Fibers. The number of neurites associated with the former junctional and extrajunctional regions of the individual muscle fibers was determined with the use of Smith interference contrast optics on a Leitz Diavert microscope. Muscle fibers in which the junctional region could be identified by AChE localization and which were not contracted in the junctional or adjacent extrajunctional regions were selected. An ocular micrometer with a $1 \mathrm{~mm}^{2}$ grid, which was subdivided into $0.1 \mathrm{~mm}^{2}$ units, was positioned over the muscle fiber so that the junctional region was to- 
Table 1. Morphological properties and synaptic molecules on short- and long-term denervated muscle fibers prior to culture.

\begin{tabular}{|c|c|c|c|c|c|c|c|}
\hline \multirow[b]{2}{*}{ Description of fibers } & \multicolumn{2}{|c|}{ AChE } & \multicolumn{2}{|c|}{$\mathrm{AChR}$} & \multirow[b]{2}{*}{ Gelasmin $\neq$} & \multirow[b]{2}{*}{ Cross strialions } & \multirow[b]{2}{*}{ Nuciei } \\
\hline & $J^{*}$ & EJt & J & EJ & & & \\
\hline Young adult (3-5 months) & + & 0 & + & 0 & + & Distinct & Peripheral \\
\hline Aged (17-24 months) & + & 0 & + & 0 & + & Distinct & Peripheral \\
\hline \multicolumn{8}{|l|}{ Denervated } \\
\hline 2 months & + & 0 & + & 0 & + & Distinct & Peripheral \\
\hline 7 months & + & Few§ & + & Few & Few & Distinct & Peripheral \\
\hline 17 months & + & Some\|l & + & Many & - & Indistinct & Some central \\
\hline 24 months & Few & Many & - & Most\# & - & Indistinct & Most central \\
\hline $\begin{array}{l}* J=\text { junctional. } \\
+E J=\text { extrafunctional. } \\
\neq \text { Gelasmin was found only in th } \\
\text { SFew fibers in the culture have } \\
\text { II Some fibers have this charact } \\
\text { "Many fibers in the culture have } \\
\text { \#ost fibers in the culture have }\end{array}$ & $\begin{array}{l}\text { tional } \\
\text { buate } \\
\text { but } m\end{array}$ & $\begin{array}{l}\text { except } \\
\text { not }\end{array}$ & & ere it $w$ & is $J$ and so & S also EJ. & \\
\hline
\end{tabular}

tally enclosed within a unit. All of the $0.1 \mathrm{~mm}^{2}$ units along the entire length of the muscle fiber were then counted by placing the edge of the grid against the side of the fiber initially contacted by neurites from the explant. The whole length of the fiber, including the junctional region, was assayed. The fibers ranged in length from 1.8 to $3.1 \mathrm{~mm}$. The distance from the center of the explant to the center of the muscle fiber was also measured.

Comparisons cited are among fibers within $20^{\circ}$ of the perpendicular to the main vector of the centrally located explant. Fibers that were more than $20^{\circ}$ from the perpendicular were not counted. Neurites in junctional or extrajunctional "units" of the grid were counted by first focusing on the plane of the upper surface of the muscle fiber. Focusing and neurite counting were continued until the lower surface of the muscle fiber was reached. Total distance between upper and lower fiber surfaces ranged from 45 to $55 \mu \mathrm{m}$. Neurites assigned to the synapse itself in the junctional region were those in the focal plane of the endplate; endplates essentially filled the $0.1 \mathrm{~mm}^{2}$ unit. Cultures were removed for fixation and processing at 24-hour intervals.

Statistics. Statistical analysis was performed using the Kruskal-Wallace ( $\mathrm{K}$ sample) test for nonparametric number distributions, $P \leq 0.05 .{ }^{23}$ Young adult and aged muscle cultures in the absence or presence of nerve were compared at similar time points to determine whether the presence of neurites near muscle fibers influenced the distribution of these molecules (J.C. Jay and K.F. Barald, "Maintenance of neurite contacts at junctional regions of cultured individual muscle fibers from aged rats is correlated with the presence of a synapse-associated protein, gelasmin," Mechanisms of Aging and Development 1989, in press).

Table 2. Morphological properties and synaptic molecules on short- and long-term denervated muscle fibers in culture.

\begin{tabular}{|c|c|c|c|c|}
\hline Description of fibers & $V$ Vability $(\%)$ & Cross striations & Nuclei & Morphological characteristics \\
\hline Young adult (3-5 months) & 76 & Distinct & Peripheral & Same as in vivo \\
\hline Aged (17-24 months) & 71 & Distinct & Peripheral & Same as in vivo \\
\hline \multicolumn{5}{|l|}{ Denervated } \\
\hline 2 months & 63 & Distinct & Peripheral & Reduction in fiber diameter \\
\hline 7 months & 62 & Distinct & Peripheral & $\begin{array}{l}\text { Reduction in fiber diameter } \\
\text { constrictions in fibers }\end{array}$ \\
\hline 17 months & 51 & Indistinct & Some central & $\begin{array}{l}\text { Reduction in fiber diameter } \\
\text { constrictions in fibers }\end{array}$ \\
\hline 24 months & 33 & Indistinct or absent & Most central & $\begin{array}{l}\text { Reductions in fiber diameter } \\
\text { constrictions in fibers }\end{array}$ \\
\hline
\end{tabular}

"Percent of viable fibers in the culture. 


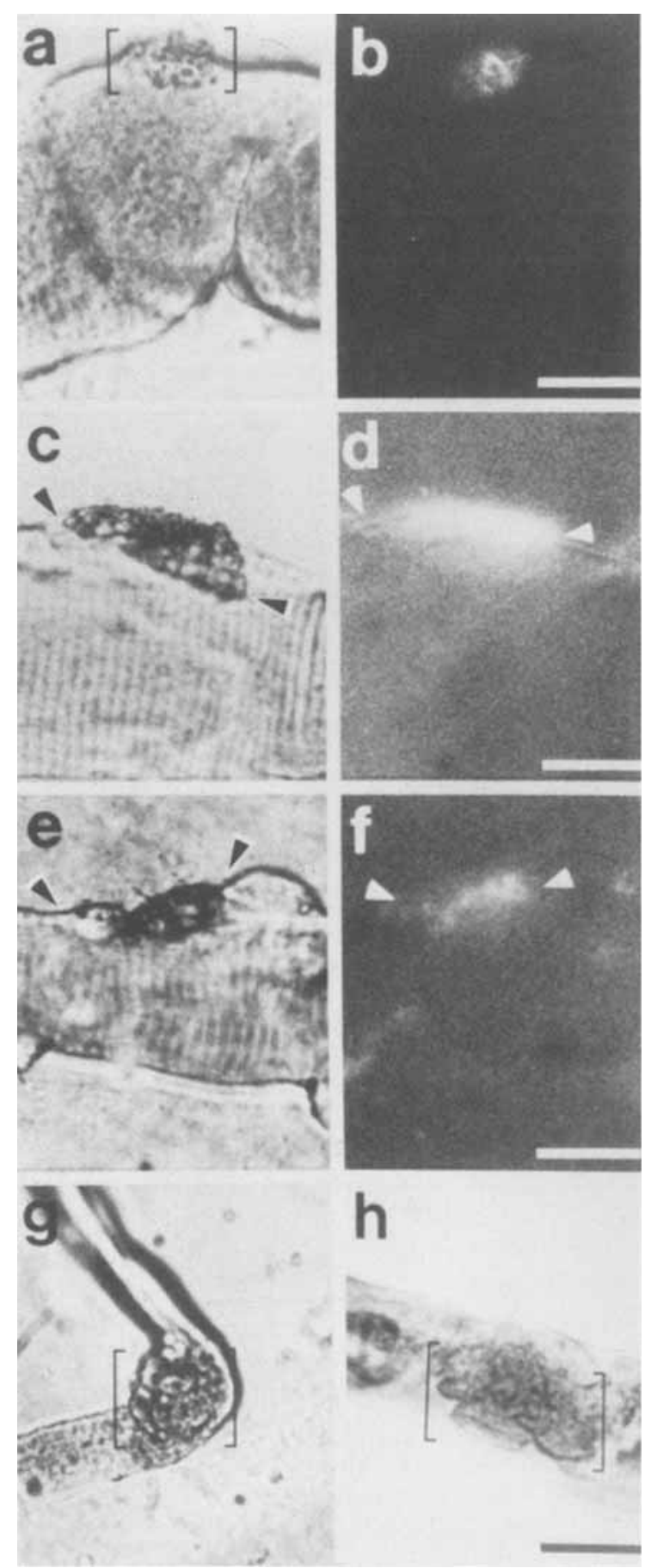

FIGURE 1. Comparison of AChE and AChRs at synaptic regions on young adult, aged, and short- and long-term denervated muscle fibers at 4 days in culture. Although $A C h E$ generally persisted at endplates, R-BTX fluorescence was dim and covered less of the junctional region on young adult and 2-5month denervated fibers. In contrast, aged muscle fibers cultured for 4 days maintained R-BTX fluoresence. (a) Four-day cultured young adult muscle fiber. Note AChE at endplate (brackets). (b) Same fiber as in a, R-BTX fluorescence. (c) Four-day cultured aged muscle fiber. Note AChE at the synaptic region (between arrows). (d) Same fiber as in c. Note R-BTX fluorescence (between arrows) corresponds to, and is as large as, the area covered by AChE stain in c. (e) Four-day cultured fiber

\section{RESULTS}

AChE, AChR, and Gelasmin on Short- and Long-Term Denervated Muscle Fibers Prior to Culture. Prior to culture, samples of undissociated muscle fibers were assayed for AChE, AChRs, and gelasmin in fresh-squash preparations of teased fibers compressed between a slide and a cover slip (see Table 1). As indicated in Table 1, aged and short-term denervated fibers (in this case denervated for 2 months) had junctional AChE, AChRs, and gelasmin comparable to young adult control fibers that were obtained from muscle in unoperated legs. With progressively longer periods postdenervation, loss of gelasmin was seen first (by $3-7$ months), then appearance of extrajunctional AChE and AChRs ( $4-7$ months), followed by loss of these molecules from the majority of endplates (17-24 months). This loss is especially notable when contrasted with the retention of all three synaptic molecules examined at the junctions of aged (24-26 months) but not denervated FDB muscle fibers.

\section{Cultures of Short- and Long-Term Denervated Muscle}

Fibers. The properties of cultured fibers from young adult animals, aged animals, and animals whose muscles were denervated for various periods of time are summarized in Table 2. Cultures of short- or long-term denervated muscle had fewer viable fibers than those prepared from young adult or aged muscle, especially after 5 months of denervation. Photographs of representative fibers from these cultures can be seen in Fig. 1. The general observations from these comparative experiments are as follows. (1) The culture environment accelerates the appearance of extrajunctional AChE and AGhR (Fig. 2) and subsequently speeds the loss of these molecules from endplates, except those of aged muscle fibers. (2) Culturing the fibers hastens the loss of gelasmin from endplates of all muscle fibers except those of

\footnotetext{
from muscle that had been denervated for 5 months prior to culture. The endplate area (between arrows) has AChE stain. (f) Same fiber as in e, R-BTX fluorescence. Note that a smaller area (between arrows) than that covered by AChE in e has AChRs. ( $g$ and $h$ ) Muscle denervated for 24 months in vivo after 1 day in culture. AChE stain was less intense compared with that on the young adult, aged, and short-term denervated fibers above. Areas of R-BTX binding sites that were colocalized with AChE but smaller than endplates (brackets) had extremely dim R-BTX fluorescence (not shown); (g) several nuclei remain at the endplate. (h) The remnants of postsynaptic folds on a fiber similar to that in $\mathbf{g}$ were outlined by AChE stain but had no AChR. Bars $=20 \mu \mathrm{m}$.
} 


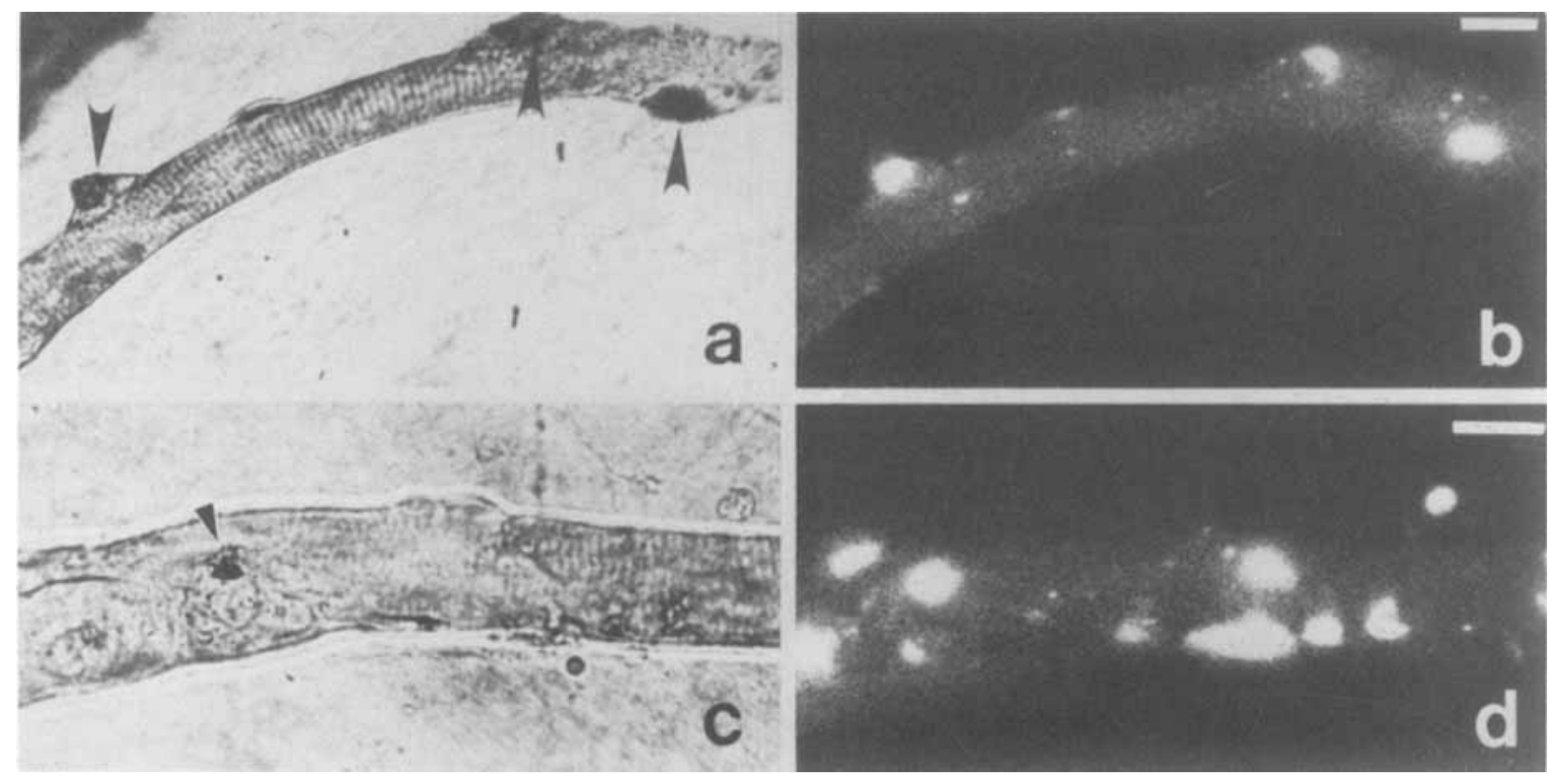

FIGURE 2. Extrajunctional AChRs and AChE on short-term denervated muscle fibers. (a) Four-day cultured fiber from muscle denervated 4 months prior to culture. AChE activity (arrows) was localized at areas of myonuclei that bulged from the muscle fiber surface. (b) The same fiber as in a. Areas of R-BTX fluorescence were colocalized with AChE patches seen in a. (c) Another muscle fiber from the same culture as the muscle fiber in a. A small area of AChE stain (arrow) was not colocalized with a myonucleus. (d) Same fiber as in c. R-BTX binding sites were not colocalized with AChE on this fiber. Extrajunctional AChRs were observed as patches of fluorescence that were variable in size and shape. Bars $=20 \mu \mathrm{m}$.

aged muscle which retain all of the three junctional molecules. In muscle denervated for $17-24$ months these molecules have, in general, been lost prior to culture (see above and Table 1). (3) After 7 months or more of denervation, morphological abnormalities were also seen in muscle fibers ( $\mathrm{Ta}$ ble 2 and Fig. 1). Some very long-term denervated fibers had only faint outlines of postsynaptic folds (Fig. 1h). Occasionally, a cluster of from four to eight nuclei was also found in this area (Fig. lg). Compare the well-defined structure and size of endplates on cultured aged fibers to the less-structured junctional regions on short- and long-term denervated cultured muscle (Fig. 1).

The decreasing ability of cultured muscle fibers to maintain endplate AChE and AChRs was related to the length of time muscle was denervated in vivo. All 2-5-month (short-term) denervated fibers had both junctional AChE and AChRs when placed in culture at 0 hour, whereas $61 \pm 7 \%(\mathrm{SEM})$ of $6-7$-month and only $8 \pm 2 \%$ of 17-24-month denervated fibers had colocalized AChE and AChRs at the NMJ. After 4 days in culture, although $74 \pm 4 \%$ of young adult fibers had junctional AChE, with increasing time after denervation in vivo, the number of fibers with junctional AChE decline; $61 \pm 7 \%$ of $6-7$-month denervated fibers retained junctional $\mathrm{AChE}$, but only $34 \pm 7 \%$ of the fibers in 17-24-month den- ervated muscle cultures retained this molecule. This was only half the number seen in control young adult cultures. On the other hand, in cultures of muscle from aged animals (26 month), 98 $\pm 1 \%$ of the fibers had junctional AChE. Extrajunctional $\mathrm{AChE}$ and $\mathrm{AChRs}$ also appeared on short-term denervated fibers (Fig. 2). Even with increasingly longer periods of denervation, junctional $\mathrm{AChE}$ persisted on more fibers than did junctional AChRs (Fig. 3).

Gelasmin pesisted at junctions of young adult muscle and aged muscle throughout the 5 day culture period, but was generally absent from junctional regions of muscle fibers denervated over 4 months. Only $35 \%$ of muscle fibers denervated 6-7 months had gelasmin at their endplates. Only $3 \%$ of fibers denervated for 24 months had gelasmin at their endplates (see Figs. 4 and 5).

\section{Comparison of Short- and Long-Term Denervated Muscle Cultures with Aged Muscle Cultures. We} attempted to determine which of the deleterious changes in endplate moleculcs that we observed in vivo and in culture were attributable to denervation and which to aging, especially in comparisons of 24-month denervated muscle with muscle from comparably aged but not denervated animals. The general observations in these studies 
A. Distribution of AChE on Short- and Long-
Term Denervated Muscle Fibers (Day 1)

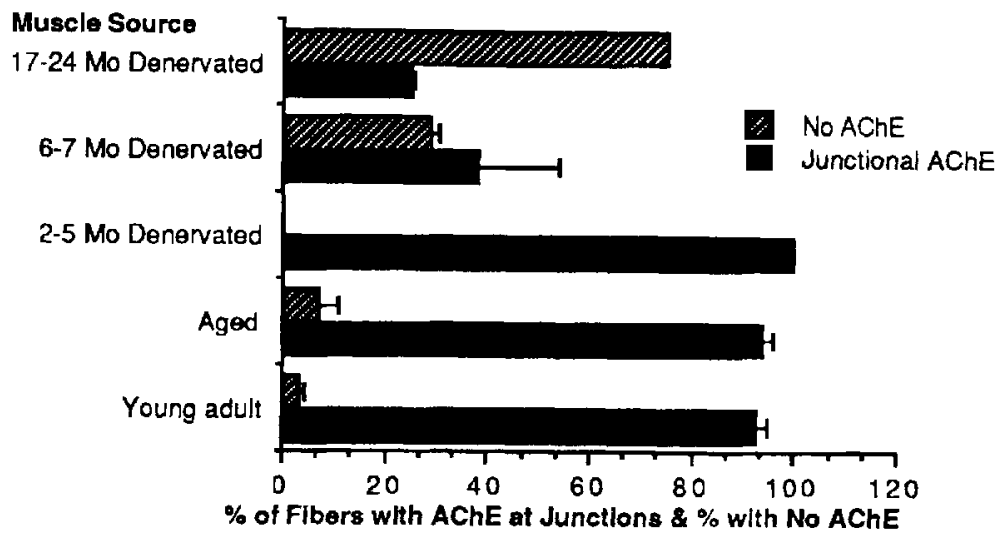

B.

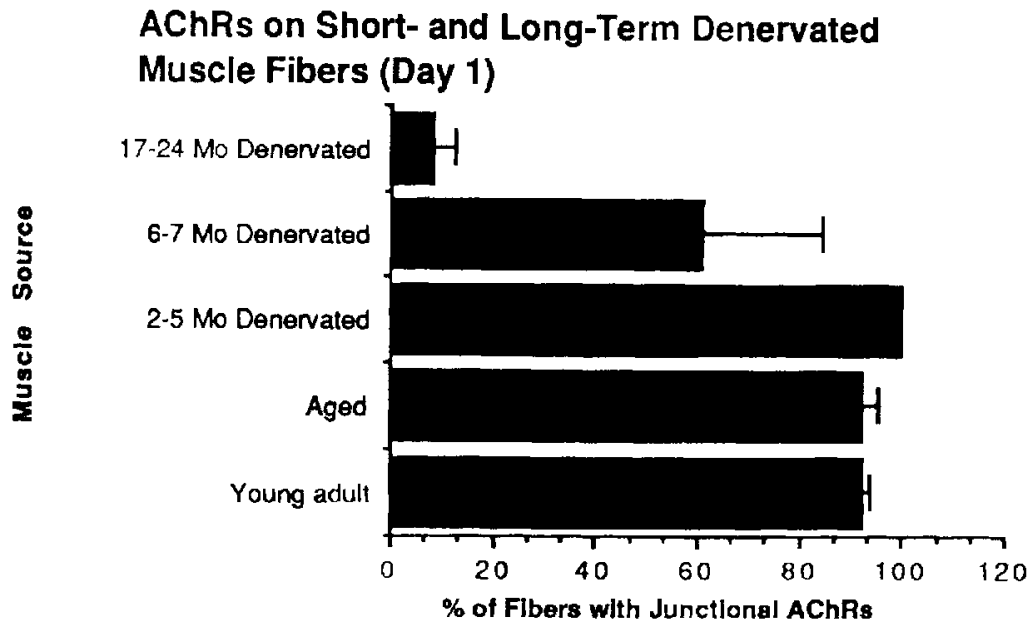

FIGURE 3. AChE and AChRs on muscle fibers in culture at day 1 in the absence of spinal cord explants. (A) The percent of fibers with junctional AChE (solid bars) or no AChE (shaded bars) are illustrated; note that the number of fibers without $\mathrm{AChE}$ at the junction increases markedly with time of denervation. (B) The percent of fibers with junctional AChRs. Note that these fibers decrease with increasing times of denervation.

(Figs. 2-7) are that the loss of junctional molecules is correlated with denervation but not aging.

Neurite Interactions with Cultured Fibers. Interactions of neurites with young adult, aged, and short- and long-term denervated fibers is illustrated in Figs. 6 and 7. Initially, spinal cord neurite outgrowth was similar in all types of nerve muscle cocultures. There was no evidence that the length of time of denervation affected initial outgrowth of neurites from the explants in muscle fiber cultures. However, although neurite contacts on young adult fibers, aged fibers, and short-term denervated fibers (up to $5-6$ months) were profuse on days $1-2$ in both the junctional and extrajunctional regions of these fibers (Fig. 6), this was not true of long-term denervated fibers. Neurites in cultures of muscle prepared from muscles denervated 6 months or more grew above and below the long-term denervated muscle fibers, but few made contact, cven at early times (days $I-2$ ), in eilther the junctional or extrajunctional regions of the fibers (Fig. 6).

Neurite contacts in the former junctional regions persisted longer than extrajunctional contacts on young adult, aged, and short-lerm denervated fibers (Fig. 6). In these studies, essentially all of the neurite contacts that persisted through the 5-day culture period were found in the former junctional region, which could be identified morphologically by the presence of synaptic folds and/ or localized AChE, except that more EJ contacts remained on aged fibers.

In cultures of long-term denervated fibers, however, two striking differences were found. 


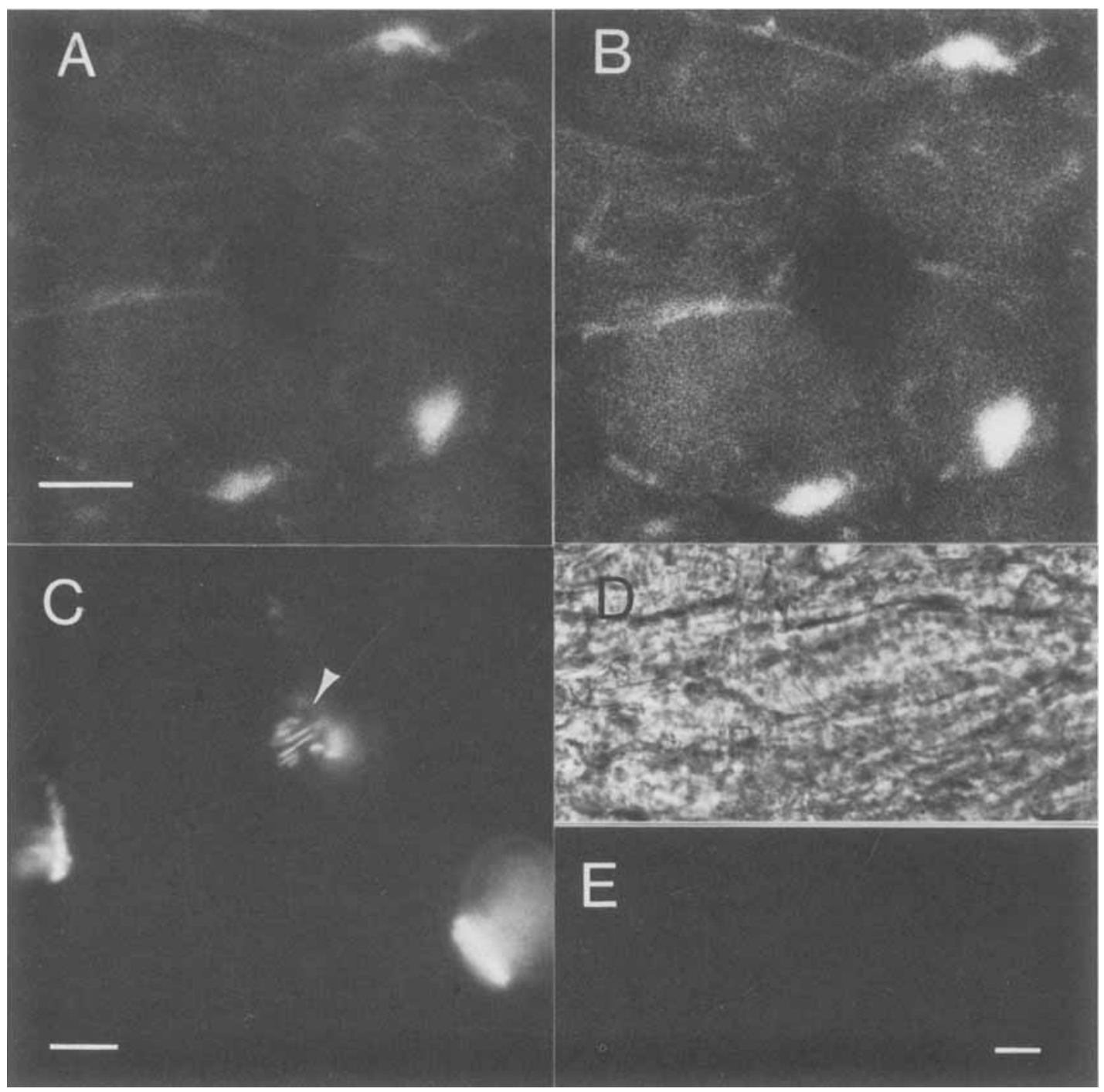

FIGURE 4. Gelasmin on young adult, aged, and long-term denervated muscle fibers (obtained from a 26-month-old rat whose muscle was denervated 24 months prior to culture). ( $\mathbf{a}$ and $\mathbf{b}$ ) A young adult FDB muscle from a 7-month-old rat was cross-sectioned through the junctional areas of several muscle fibers; in a the fibers are stained with R-BTX for AChR, and in $\mathbf{b}$ they are stained with the monoclonal antibody to gelasmin, SBL-1, and a second step rabbit antimouse IgG antibody conjugated with fluorescein. Fibers were viewed with the appropriate epifluorescence optics. Note the colocalization of the AChR and gelasmin on these fibers. (c) A fresh squash of aged (24 months) FDB muscle fibers prepared as described in the text was stained for gelasmin with the monoclonal antibody SBL-1 and the second step antibody as described in b. Note the elaborate neuromuscular junction indicated by the arrow; compare this junction with the large junction seen in Fig. 1a. (d) A fresh squash of long-term (17 month) denervated muscle is seen. This preparation has been stained with the modified Karnovsky procedure for AChE, and several small deposits can be seen as concentrations of black spots. However, no gelasmin is seen on these fibers (e) after staining with SBL-1 and the appropriate second step antibody. No AChRs were present on these fibers (not shown). Bars $=50 \mu \mathrm{m}$.

Numbers of contacts at all times in both junctional and extrajunctional regions of the muscle fibers were far fewer than those in any other type of culture, including those from comparably aged but not denervated muscle fibers (Fig. 6). In addition, there was little difference between numbers of contacts seen in junctional versus extrajunctional areas with time, although consistent differences between numbers of contacts in these two regions were seen in all other types of muscle fiber cultures, including aged muscle fibers.

\section{Correlation of Contact Points with Gelasmin.}

When the contact points of all the cultures were examined for the three "synapse-specific" molecules examined in this study, another striking difference was noted. The presence of AChE and AChRs at contact points was variable in all cul- 


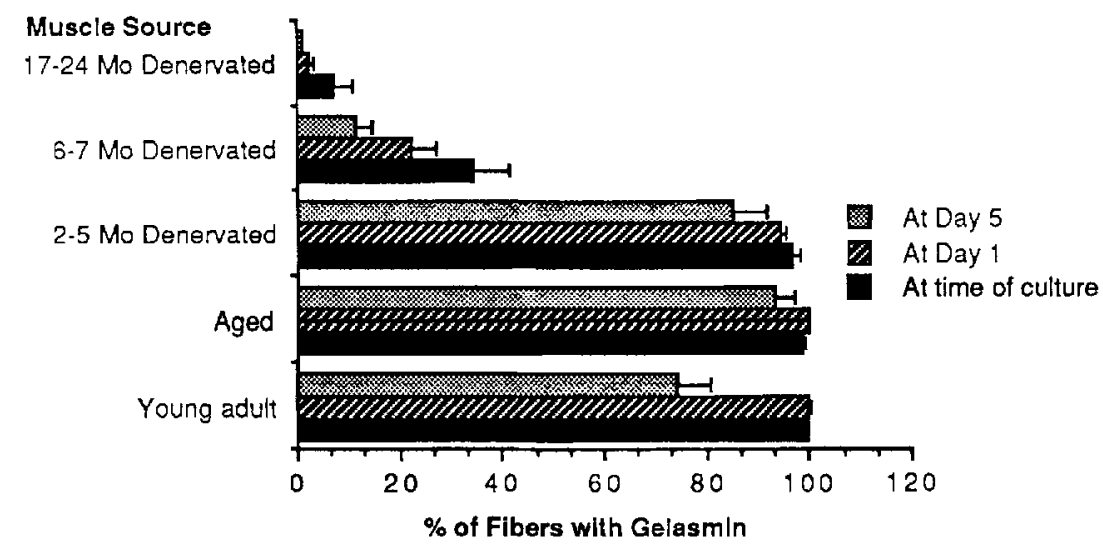

FIGURE 5. Comparison of gelasmin distribution on fibers just prior to culture and after 1 and 5 days in culture.

Neurte contacts on young adult muscle flbers

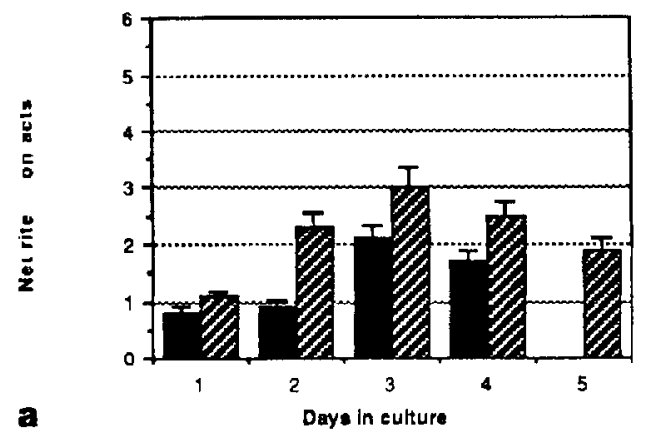

Neurite contacts on 2-5 month denervated muscle fibers

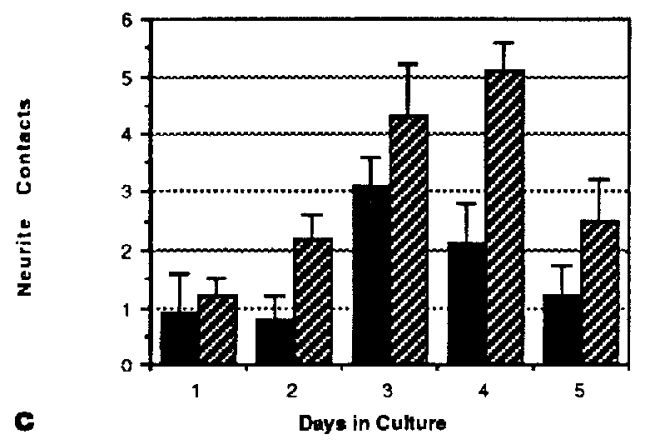

Neurite contacts on aged muscle flbers

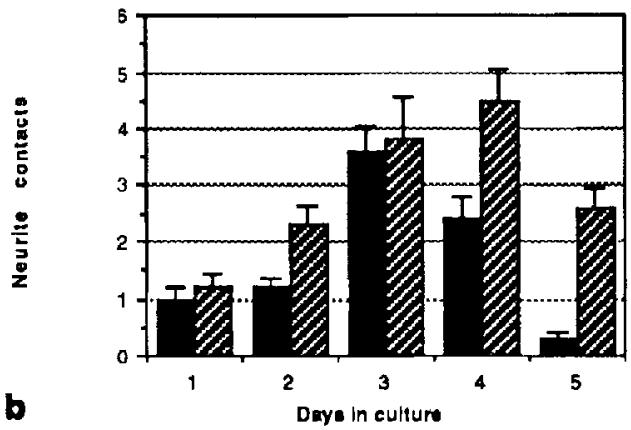

Neurite contacts on 6-7 month denervated muscle flbers

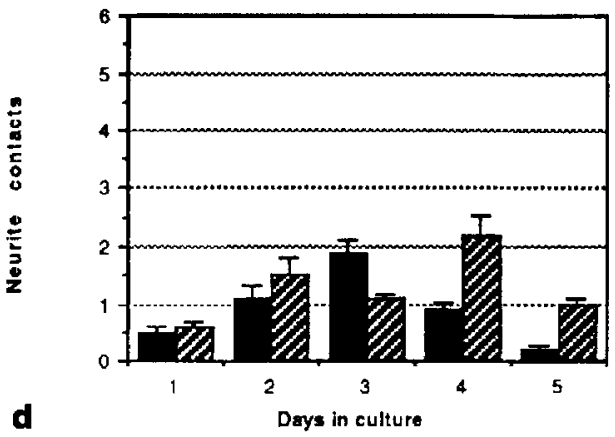

Neurite contacts on 17-24 month denervated muscle fibers

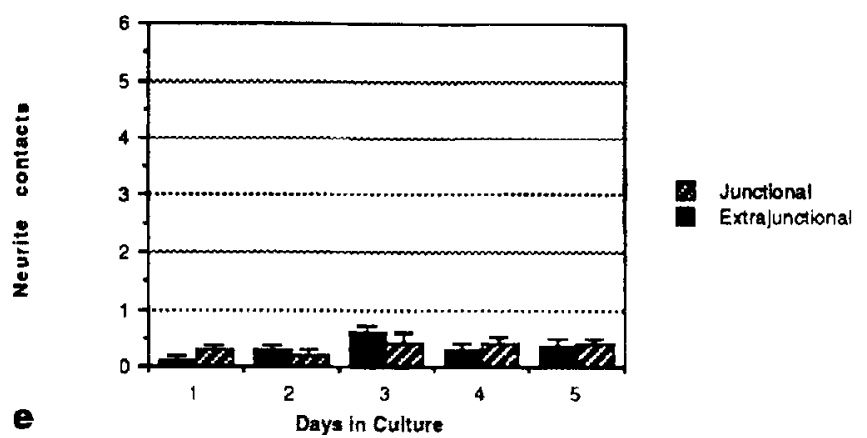

FIGURE 6. Distribution of neurite contacts on (a) young adult fibers in the junctional and extrajunctional regions, (b) aged fibers, (c) 2-5-month denervated fibers, (d) 6-7-month denervated fibers, and (e) 17-24-month denervated fibers. 

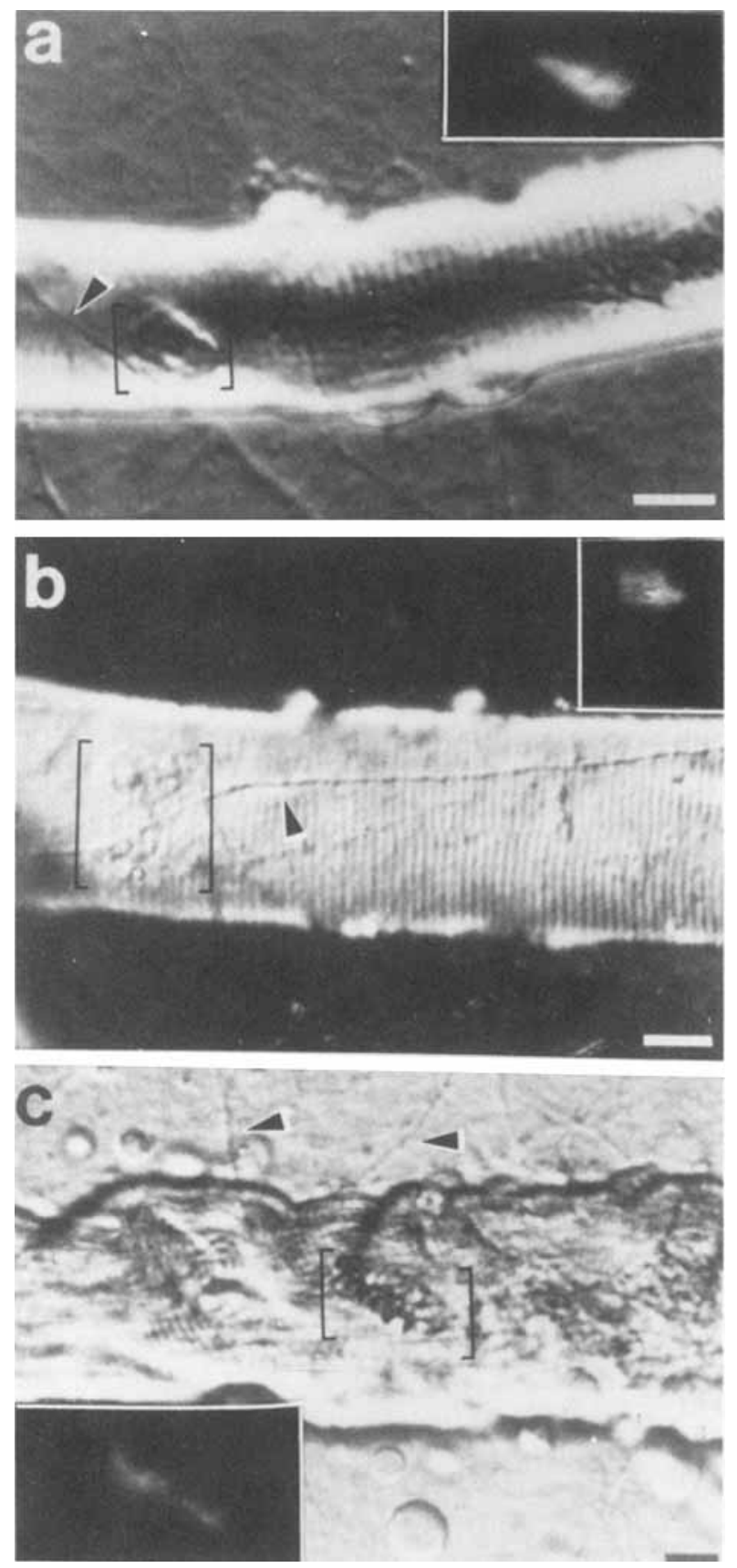

FIGURE 7. Neurite interaction with young adult, aged, and longterm denervated muscle fibers as viewed with Smith interference optics and subsequent staining of the fibers for gelasmin with the monoclonal antibody SBL-1 as described in the text. Spinal cord explants were added to cultures containing muscle fibers that had been in culture for 1 day. (a) Young adult muscle fiber. A neurite (arrow) was observed crossing the muscle fiber surface near the endplate (brackets). This 3-day nervemuscle culture contained explants that had been present for 2 days. Insert: gelasmin staining at the junction viewed after staining with SBL-1 and a fluorescein-conjugated rabbit antimouse second step antibody viewed with appropriate epifluorescence optics for fluorescein. (b) Aged muscle fiber. A neurite (arrow) traveled close to the junctional region (brackets). This 5-day nerve-muscle culture contained explants that had been present for 4 days. Insert: the presence of gelasmin, re- tures, regardless of the history of the muscle fibers. Although the number of neurite contacts that were maintained in junctional regions of muscle fibers from young adult, aged, and shortand long-term denervated muscle were inversely proportional to the length of denervation time, we found that gelasmin was present at all sites of neurite contact regardless of the age or denervation history of the muscle fibers (Fig. 7). Gelasmin was present even in the absence of $\triangle \mathrm{ChE}$ (Fig. 7). It was also present at a few junctional regions that were not contacted by neurites in all of the cultures. The number of fibers with gelasmin in long-term denervated cultures was far fewer than in any other culture type (Fig. 7), although gelasmin was present on those very few fibers that had nerve contacts (Fig. 7). Gelasmin was present at former junctional regions. When muscle is denervated for long periods of time, gelasmin disappears from the former $\mathrm{NMJ}$; this disappearance is correlated with the altered nerve-muscle interactions seen in culture.

\section{DISCUSSION}

One important finding of these studies is that the denervated FDB culture model mirrors the events seen in vivo after denervation of adult rat muscle. In general, we saw effects on AChE and AChRs that are in accord with previous findings of others in vivo and in vitro. Our finding, that AChE persists at the NMJ of both freshly isolated and cultured FDB fibers longer than AChRs, agrees with observations that AChE remains longer than AChRs at denervated endplates in the cat and frog. ${ }^{12,24}$ Although the appearance of extrajunctional AChE and AChRs does not occur as rapidly in the FDB as in other muscles in vivo (see the section on muscle fibers prior to culture), their loss is correlated with length of denervation in vivo. The alterations at the molecular level that are responsible for loss of acetylcholine receptors before disappearance of $\mathrm{AChE}$ from endplates are presently not known. However, these changes are clearly enhanced by progressively longer periods

\footnotetext{
vealed by SBL-1 staining at the junction. (c) Six-month denervated fiber. Neurites (arrows) were observed traveling above or below the muscle fiber surface, but many fewer neurites make contact with such muscle fibers. This nerve-muscle culture was 5 days old. $\mathrm{AChE}$ was present at the junctional region (brackets); gelasmin staining was seen at the junction (insert). Endplates on all fibers were located by the presence of raised postsynaptic folds and/or AChE prior to localization of gelasmin. Bars $=20 \mu \mathrm{m}$
} 
of denervation and are also accelerated in culture, making the culture model an especially useful tool for the study of the mechanism(s) of their disappearance. The general conclusion of these experiments is that the presence of $\mathrm{AChE}$ and AChRs at nerve-muscle contact points is variable; therefore, these molecules are apparently not good candidates as molecular cues for reinnervation.

The results of these studies are, however, consistent with the possibility that gelasmin, or some similar molecule, may serve as a reinnervation clue for a returning nerve and that reinnervation may be dependent on its presence. We found that the synapse-enriched $93 \mathrm{kD}$ glycoprotein, gelasmin, which we have studied extensively, ${ }^{1,2}$ was present at all sites of neurite contact in cultures of young adult, aged, and short- and longterm denervated muscle alike. Furthermore, the presence of gelasmin was well correlated with the success of neurite-muscle contact formation and persistence in these cultures. Gelasmin was present on some muscle fibers, even long-term denervated muscle fibers, in cultures without neurites. We believe from previous studies ${ }^{1,2}$ as well as these studies that gelasmin is a product of the muscle and not the nerve. Its loss from denervated fibers probably reflects the need for either trophic or electrophysiological neural input of some type.

Gelasmin may not be the sole or even the primary molecule responsible for stabilizing neurite contacts, however. Some as yet unidentified molecule, with which gelasmin may or may not be associated, could be more important for reinnervation. Furthermore, we have not ruled out the possibility that other molecules, including AChE and AChRs or a myriad of other synapse-specific molecules, ${ }^{20-22}$ may also be involved in major or minor roles.

In view of the observation that 6 months or more of denervation prior to culture, but not agerelated changes, are associated with the decreasing ability of cultured muscle fibers to retain AChE, AChRs, and gelasmin, what conclusions might be drawn about the persistence of molecular cues that could be important for reinnervation? Molecules that may be important for reinnervation may not be greatly affected by age-related changes at the majority of synapses or by a short time without nerve ( $2-5$ months). ${ }^{9}$

A recent report ${ }^{7}$ on aging rat neuromuscular junctions, however, indicates that extensive remodeling of the NMJ goes on throughout life and that aged junctions are more likely to be found denervated in vivo than young junctions, at least in soleus muscle. Soleus muscle, however, does not receive constant use in old animals, and the case is apparently different with a constantly used muscle, such as the diaphragm, which exhibits little remodelling. ${ }^{7}$ Denervation of aged muscle may be a consequence of the loss or alteration of some basal laminal or plasmalemmal component(s) associated with maintenance of synaptic contacts, but these components are unlikely to be either AChRs or AChE. Longer periods of denervation probably result in the loss of molecules such as gelasmin that could be important for nerve-muscle recognition and synaptogenesis as well as changes in AChE and AChRs. Other biochemical and/or structural changes in longterm denervated muscle are, no doubt, also related to the decreased ability of muscle fibers to accept reinnervation. Although factors that remain at the original endplate for perhaps a few weeks or months after denervation may be important for reinnervation, in rats the extrajunctional region must not be excluded as a possible site for synapse reformation. In rat muscle, after a short period of denervation, ectopic synapses may form on muscle that also had original endplates that are apparently available for reinnervation. ${ }^{26}$ In other studies, we have shown that low levels of gelasmin are present in extrajunctional regions, ${ }^{1,2}$ and thus the molecule could serve as a potential guide for the formation of ectopic synapses as well, such as those on aged muscle.

How then is the state of the muscle fiber related to the site of reinnervation after various periods of denervation? What governs the choice of reinnervation at the old endplate versus ectopic regions, or why does nerve fail to reinnervate in some cases? We presently do not have answers to these questions, but such questions are potentially addressable in a tissue culture system. The role of factors associated with muscle synaptic and/or extrasynaptic regions in mammalian reinnervation can be investigated in such a system, and we can separate muscle-derived factors or structural elements from other nonmuscle contributions to the success or failure of reformation of nervemuscle connections, such as connective tissue and fat deposition and the availability of nerve for reconnection. Additional factors such as aging or length of denervation time in vivo can also be studied to determine the role of muscle-associated molecules that are important in the reinnervation process. 


\section{REFERENCES}

1. Barald KF, Phillips GD, Jay JJ, Mizukami IF: A component in mammalian muscle synaptic basal lamina induces clustering of acetylcholine receptors. Prog Brain Res 71:397408 .

2. Barald KF, Phillips GD, Jay IJ: Gelasmin: an acetylcholine receptor clustering factor derived from mammalian muscle extracellular matrix: I. Location on embryonic rat myotubes and at adult synapses. Development, in press.

3. Bennett MR, Pettigrew AG: The formation of synapses in striated muscle during development. J Physiol (Lond) $1974 ; 241: 515-545$.

4. Boeke J: Nerve endings, motor and sensory, in Penfield W (ed): Cytology and Cellular Pathology of the Nervous System (fascimile of the 1932 edition). New York, Hafner Press, 1965, vol 1, pp 243-315.

5. Brown MC, Hopkins WG: Role of degenerating axon pathways in regeneration of mouse soleus motor axons. $I$ Physiol (Lond) 1981;318:365-373.

6. Cardasis CA: Ulirastructural evidence of continued reorganization at the aging $(11-26 \mathrm{mo})$ rat soleus neuromuscular junction. Anat Record 1983;207:339-415.

7. Cardasis CA, LaFontaine DM: Aging rat neuromuscular junctions: a morphometric study of cholinesterase-stained whole mounts and ultrastructure. Muscle Nerve 1987 10:200-213.

8. Goldmann EE: Die aussere und innere sekretion des gesunden organismus im lichte der vitalen farbung. Beitr Klin Chir 1909;64:192-265.

9. Gorio A, Carmignoto G, Finesso M, Polato P, Nunzi MG: Muscle reinnervation. II. Sprouting, synapse formation and repression. Neuroscience 1983:8:403-416.

10. Gutmann E, Young JZ: The reimnervation of muscle after various periods of atrophy. J Anat 1944;78:15-57.

11. Jay J, Barald KF: Improved culture of individual muscle fibers with and without spinal cord explants in a collagen gel. I Neurosci Methods 1985; 15:229-234.

12. Krause M, Wernig A: The distribution of acetylcholine receptors in the normal and denervated neuromuscular junction of the frog. J Neurocytol 1985;14:765-780.

13. Maltin CA, Duncan L, Wilson AB: Rat diaphragm: changes in muscle fiber type frequency with age. Muscle Nerve 1985;8:211-216.

14. Marshall LM, Sanes JR, McMahan UJ: Reinnervation of original synaptic sites on muscle fiber basement membrane after disruption of the muscle celis. Proc Nat Acad Sci USA 1977;74:3073-3077.

15. Merlie JP, Iscnberg KE, Russell SD, Sanes JR: Denervation supersensitivity in skeletal muscle: analysis with a cloncd cDNA probe. I Cell Biol 1984;99:332-335.

16. Mishell BB, Shiigi SM: Selected Methods in Cellular Immunology. San Francisco, WH Freeman \& Co, 1980, pp 16-17.

17. Moody-Corbett F, Cohen MW: Localization of cholinesterase at sites of high acetylcholine receptor density on embryonic amphibian muscle cells cultured without nerve. $J$ Neurosci 1981:1:596-605.

18. Ravdin P, Axelrod D: Fluorescent tetramethyl rhodamine derivatives of alpha-bungarotoxin: preparation, separation, and characterization. Anal Biochem 1977;80:585-592, and correction 1977;83:336.

19. Redfern PA: Neuromuscular transmission in new-born rats. J Physiol (Lond) 1970;209:701-709.

20. Sanes JR: Laminin, fibronectin, and collagen in synaptic and extrasynaptic portions of muscle fiber basement membrane. J Cell Biol 1982;93:442-451.

21. Sanes JR, Covault J: Axon guidance during reinnervation of skeletal muscle. Trends Neurosci 1985;8:523-528.

22. Scott LJC, Bacou F, Sanes JR: A synapse-specific carbohydrate at the neuromuscular junction: association with both acetylcholinesterase and a glycolipid. I Neurosci 1988; $8: 932-944$

23. Siegel S: Non-parametric Statistics. New York, McGraw-Hill, 1956, pp 184-194.

24. Steinbach JH: Neuromuscular junctions and alpha-bungarotoxin-binding sites in denervated and contralateral cat skclctal muscles. J Physiol (Lond) 1981;313:513-528

25. Sunderland S: Nerves and Nerve Injuries. London, E \& S Livingstone, 1968, p 330.

26. Womble MD: The clustering of acetylcholine receptors and formation of neuromuscular junctions in regenerating mammalian muscle grafts. Am J Anat 1986;176:191-205. 